\title{
DYNAMICAL EFFECTS OF MERGERS
}

\author{
J.E. BARNES \\ Institute for Astronomy, University of Hawai' $i$ \\ 2680 Woodlawn Drive, Honolulu, HI 96822, USA
}

\section{Introduction}

The bridges and tails of interacting galaxies were elegantly explained when Toomre \& Toomre (1972) showed that such features arise from tides acting on disk galaxies. Emboldened by this success, Toomre \& Toomre proposed that certain twin-tailed fuzzballs without obvious interaction partners were in fact the merged relics of interacting pairs, and that such relics eventually become elliptical galaxies. Observations of twin-tailed systems found evidence for both their tidal origins and their elliptical destinies (e.g. Schweizer 1986), while self-consistent numerical simulations substantiated theoretical predictions of rapid orbital decay and the elliptical-like outcome of violent relaxation during merging (e.g. Barnes 1988, and references therein). But while our basic picture of merging seems solid, the precise role of this process in galaxy evolution is not so clear. Dynamical studies provide some insight into this issue; at present we can distinguish three levels of reliability in the numerical work: solid results, good bets, and hopeful guesses.

\section{Solid Results}

Confidence in numerical models may be grounded in the realization that self-consistent $N$-body simulation is essentially a Monte-Carlo technique for solving the coupled collisionless Boltzmann and Poisson equations (White 1982, Hernquist \& Barnes 1990). As with other Monte-Carlo methods, $N$ body simulations yield results with errors scaling as $N^{-1 / 2}$; the steady progress of computers enables experimenters to perform increasingly subtle calculations.

Tidal models of interacting systems can often be built by combining "offthe-shelf" components. Fig. 1 shows a self-consistent lookalike of Arp 252 produced by a parabolic encounter of two equal-mass galaxies, each con- 
sisting of a central bulge, an exponential disk, and a dark halo comprising $80 \%$ of the mass. Even without velocity data, such modeling can yield interesting insights; for example, to match the phase of the bar in the lower disk, the bulge must not be so compact and massive as to dominate the rotation curve at small radii. And as Stockton (1974) showed by checking Toomre \& Toomre's (1972) reconstruction of The Mice, models matching the appearance of interacting systems can make qualitative predictions about velocities.
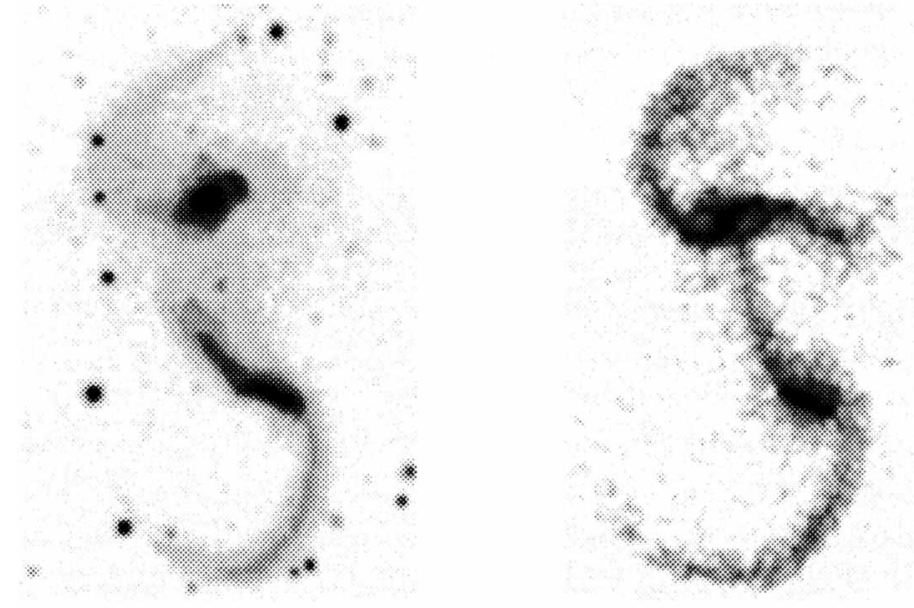

Figure 1. R-band image of Arp 252, courtesy Bill Keel (left) and a grey-scale representation of an $\mathrm{N}$-body lookalike (right). The dark halos are not shown.

Strong encounters like this one, extrapolated into the future, merge after only one or two additional orbits. Tidal interactions between dark halos are largely to blame for such rapid orbit decays (White 1978); halos efficiently absorb orbital angular momentum from the luminous material (Barnes 1988, 1992). Indeed, close-passing disks can merge well before their tidal tails disperse, creating "twin-tailed" relics like NGC 7252 (Schweizer 1982, Hibbard et al. 1994), as a self-consistent model for that very galaxy illustrates (Hibbard \& Mihos 1995).

Self-consistent simulations of equal-mass disk galaxy encounters have repeatedly produced merger remnants with some characteristics of normal ellipticals (Farouki \& Shapiro 1982, Negroponte \& White 1983, Gerhard 1983a, Barnes 1988, Hernquist 1993a). Violent relaxation due to orbit scattering by time-dependent gravitational fields tends to erase existing structures (Lynden-Bell 1967); in merger simulations, the generic result is a pressure-supported ellipsoidal hulk. And here as in other studies of violent relaxation (May \& van Albada 1984), these hulks have cores with radii de- 
termined by the peak central phase-space densities of the initial galaxies, grafted onto luminosity profiles resembling a de Vaucouleurs (1948) law at larger radii.

The gravitational field of a merger generally settles down before particle orbits are fully randomized, so traces of progenitor structure $d o$ survive the process. Radial abundance and color gradients are likely to be only moderately reduced by dissipationless mergers since initial and final binding energies are fairly well-correlated (White 1980, Barnes 1988). More detailed simulations have shown that kinematic signatures of the initial disks can also survive merging (Gerhard 1983b, Barnes 1992, Hernquist 1993a). For example, these simulated merger remnants often exhibit large misalignments between their spin and minor axes, due to streaming of particles on major-axis tube orbits (Levison 1987); on the other hand, most elliptical galaxies seem to have smaller misalignments (Franx, Illingworth, \& de Zeeuw 1991).

\section{Good Bets}

While gas-dynamical modeling does not yet inspire as much confidence as collisionless $N$-body simulation, calculations combining $N$-body and Smoothed Particle Hydrodynamic (SPH) methods are probably on the right track. SPH converges to the standard equations of motion for a compressible fluid in the $N \rightarrow \infty$ limit (e.g. Monaghan 1992, and references therein), although existing adaptive implementations do not get the gas thermodynamics quite right (Hernquist 1993b, but see Nelson \& Papaloizou 1994). In any case, the actual dynamics of the ISM are vastly more complex than those of any foreseeable numerical model. But over kpc scales, rapid cooling and momentum conservation appear to be the key ingredients required for numerical simulations; SPH and "sticky-particle" codes both produce reasonable first approximations in this regime.

One outcome seen consistently in gas-dynamical models is rapid inflows of dissipative material in tidally disturbed disks (Noguchi 1988, 1991, Hernquist 1989, Combes, Dupraz, \& Gerin 1990, Barnes \& Hernquist 1991, 1996). In a disk with of any kind of non-axisymmetric structure, gravitational torques transfer angular momentum from dissipative to collisionless components (e.g. Simkin, Su, \& Schwarz 1980, Carlberg \& Freedman 1985, von Linden et al., these proceedings). The structures produced in tidallyperturbed disks are quite effective at creating such torques, and the inflows which result may well explain the kpc-scale central starbursts seen in interacting disk systems (Bushouse 1987).

Indeed, gravitational torques and dynamical friction are so remarkably efficient at transporting spin and orbital angular momentum in dissipative 
merger simulations that about half the entire gas inventory is promptly concentrated within a central blob of radius comparable to the model's spatial resolution (Negroponte \& White 1983, Barnes \& Hernquist 1991, Noguchi 1991; also Fig. 2). Violent relaxation alone can't explain how so much gas is repeatedly scattered inward; a general although rather qualitative explanation might be that the inward scattering of gas maximizes the dissipation rate and consequently the production of entropy (Barnes \& Hernquist 1996).
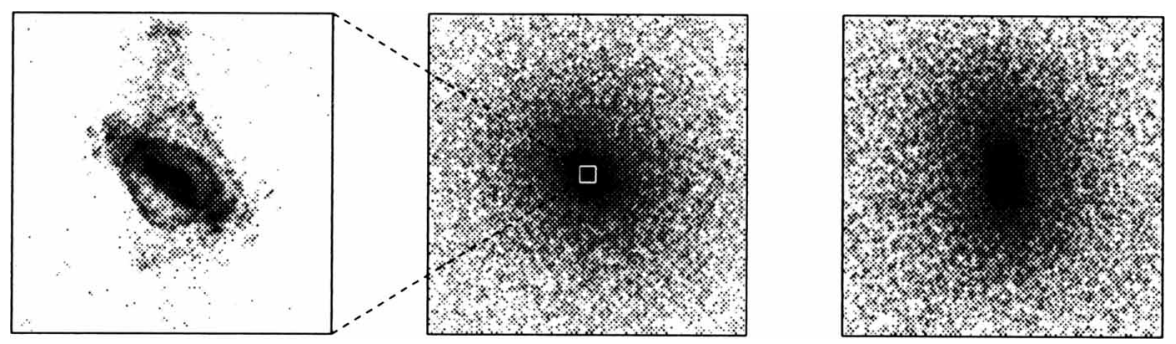

Figure 2. Nuclear gas cloud (left) in simulated merger remnant (middle), and a pure $N$-body version of the same calculation (right).

In terms of their masses and radii, these simulated gas blobs are comparable to the central clouds of molecular material detected in merger remnants like as NGC 520 (Sanders et al. 1988) and Arp 220 (Scoville et al. 1991). Although the coincidence of radii may be partly due to modeling limitations, mergers do seem capable of assembling massive central clouds on the short time-scales needed to fuel ultra-luminous starbursts (Larson 1987).

Gas driven to the center of a remnant by strongly time-dependent gravitational torques may become kinematically decoupled from the galaxy it resides in. Out of four $N$-body/SPH simulations of equal-mass encounters, one produced a striking example of such kinematic decoupling in the form of a pair of counter-rotating gas disks (Hernquist \& Barnes 1991, Barnes \& Hernquist 1996). Similar counter-rotating disks are found in the prototypical merger remnant NGC 7252 (Schweizer 1982), and the idea that such structures can evolve into kinematically-decoupled cores is supported by observations of steep metalicity gradients in such cores (Bender \& Surma 1992, Davies, Sadler \& Peletier 1993).

Detailed analysis of a few cases indicates that the central gas clouds formed in simulated remnants can markedly affect the shape of the stellar distributions of these systems, reducing overall flattening and favoring more oblate structures (Barnes \& Hernquist 1996; see also the right-hand panel of Fig. 2). A similar result has been found in cosmological simulations (Katz 
\& Gunn 1991, Urdy 1993). This effect may be due to destabilization of box orbits in the deep potential wells produced by the central gas (Dubinski 1994). If this explanation is right, there should be a correlation between the initial central concentrations of the galaxies involved and the oblateness of the remnants they produce which could be studied in pure $N$-body simulations.

Gas which returns to the merger remnant at later times has too much angular momentum to reach the center; this material forms a warped disk extending to several effective radii (e.g. Barnes \& Hernquist 1986). Similar disks are also seen in early-type galaxies like NGC 4753 (Steiman-Cameron, Kormendy \& Durisen 1992). Star formation in well-settled gas disks could produce the vestigial stellar disks found in some elliptical galaxies (e.g. Bender 1988, Scorza \& Bender, these proceedings).

\section{Hopeful Guesses}

Finally, there are some topics which are as yet mostly matters of conjecture. While it is not clear what role merging plays in galaxy evolution, it would be very puzzling if merger remnants were shown to evolve into objects categorically unlike real galaxies!

Star formation is at the core of many unsolved problems concerning effects of mergers. To begin with, there is the worry that star formation or subsequent supernovae might deplete or disperse the interstellar material before it can form clouds like the one shown in Fig. 2. Simulations are not a very effective way to address this worry, since star formation can only be crudely included; it may be more productive to use observations of such gas clouds in luminous IR galaxies (Scoville et al. 1991) to constrain the disruptive effects of star formation. A more subtle concern involves the timing of starbursts; here calculations show that the rate at which highdensity gas accumulates is sensitive to the encounter parameters (Barnes \& Hernquist 1996) and to the internal structure of the galaxies involved (Mihos \& Hernquist 1994a). At present it is not clear if most merging galaxies go through brief but very intense starbursts, or if only mergers with rather special initial conditions attain high rates of star formation (Hibbard 1995).

A related worry concerns the distribution of the stars produced in a merger-induced starburst. Numerical simulations with star-formation rules have produced remnants with pronounced central luminosity spikes (Mihos \& Hernquist 1994b). Such spikes are not evident in real elliptical galaxies; to be consistent with the observations, nuclear starburst populations should join smoothly onto the bodies of galaxies, without terribly obvious discontinuities in luminosity, color, or abundance. It is quite possible that 
feedback mechanisms not yet included in the simulations may be involved in matching nuclear starburst populations onto the galaxies they inhabit; the need for similar feedback mechanisms has long been recognized in hierarchical models for galaxy formation (White \& Rees 1978; see Kaufmann, these proceedings).

The focus on mergers of equal-mass galaxies in existing simulations is partly due to computational limitations; unequal-mass encounters take longer to merge and require larger $N$ to reveal more subtle effects. Shown in Fig. 3 is the result of a close retrograde passage of two disk galaxies with a 3:1 mass ratio; the larger disk has survived the merger - albeit with considerable heating - because of its relatively weak response to its retrograde companion. In a small sample of such 3:1 mergers, disk-like kinematics persist in about half of the remnants produced. This suggests that disk galaxies may sometimes accrete small companions without suffering too much heating. Yet mass ratios of 10:1 or more, which are probably more relevant for typical disk galaxies (Toth \& Ostriker 1992), are still largely beyond the reach of systematic numerical studies (Walker, Mihos \& Hernquist 1995).
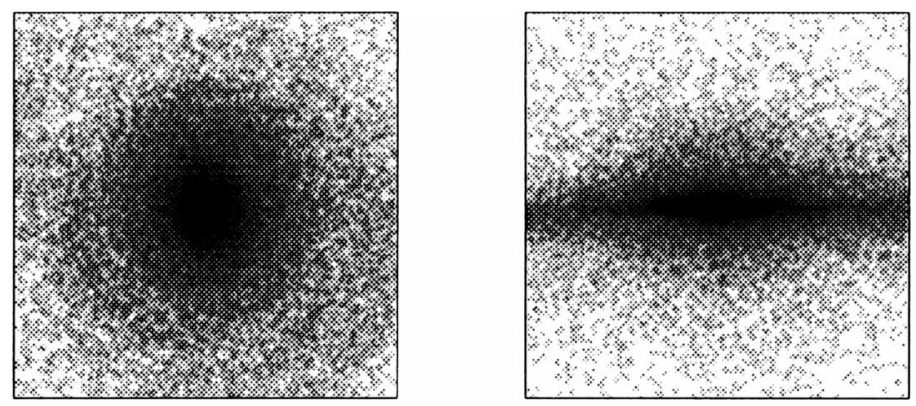

Figure 3. Face-on (left) and edge-on (right) views of an S0-like merger remnant produced by a 3:1 retrograde encounter.

Another topic yet scarcely touched concerns multiple mergers in groups and small clusters. It seems reasonable that such mergers should tend to smear or average out the kinematic signatures of individual disks, and this expectation is supported by the few simulations which have been performed (Barnes 1989, Weil \& Hernquist 1994). It also seems likely that dissipationless mergers would disrupt the kinematically decoupled cores seen in many elliptical galaxies, although simulations have not yet been done to check this point. If multiple mergers do play an important role in the formation of cluster ellipticals, decoupled cores should be rare in such galaxies.

I thank Lars Hernquist for permission to discuss our unpublished work, and Frank van den Bosch and Susanne von Linden for help with the slides and 
video for this talk. The Pittsburgh Supercomputing Center and the Maui High Performance Computing Center provided computing time for this research. I acknowledge partial support from NASA grant NAG 5-2836.

\section{References}

Barnes, J.E. 1988. Ap.J. 331, 699.

Barnes, J.E. 1989. Nature 338, 123.

Barnes, J.E. 1992. Ap.J. 393, 484.

Barnes, J.E. \& Hernquist, L. 1991. Ap.J. 370, L65.

Barnes, J.E. \& Hernquist, L. 1996. Ap.J., in press.

Bender, R. 1988. $A \& A$ 202, L5.

Bender, R. \& Surma, P. 1992. $A \& A$ 258, 250.

Bushouse, H.A. 1987. Ap.J. 320, 49.

Carlberg, R. \& Freedman, W.L. 1985. Ap.J. 298, 486.

Combes, F., Dupraz, C., \& Gerin, M. 1990. In "Dynamics and Interactions of Galaxies", ed. R.Wielen (Springer: Berlin), p. 205.

Davies, R.L., Sadler, E.M., \& Peletier, R.F. 1993. MNRAS 262, 650.

de Vaucouleurs, G. 1948. Ann. d'Astrophys. 11, 247.

Dubinski, J. 1994. Ap.J. 431, 617.

Farouki, R.T. \& Shapiro, S. 1982. Ap.J. 259, 103.

Franx, M., Illingworth, G.D., \& de Zeeuw, T. 1991. Ap.J. 383, 112.

Gerhard, O.E. 1983a. MNRAS 202, 1159.

Gerhard, O.E. 1983b. MNRAS 203, 19P.

Hernquist, L. 1989. Nature 340, 687.

Hernquist, L. 1993a. Ap.J. 409, 548.

Hernquist, L. 1993b. Ap.J. 404, 717.

Hernquist, L. \& Barnes, J. 1990. Ap.J. 349, 562.

Hernquist, L. \& Barnes, J. 1991. Nature 354, 210.

Hibbard, J.E. 1995. PhD Thesis, Columbia University.

Hibbard, J.E. et al. 1994. A.J. 107, 67.

Hibbard, J.E. \& Mihos, J.C. 1995. A.J. 110, 140.

Katz, N. \& Gunn, J.E. 1991. Ap.J. 377, 365.

Larson, R.B. 1987. In "Starbursts and Galaxy Evolution", eds. T.X. Thuan, T. Montmerle, J.T.T. Van, (Editions Frontieres: Gif sur Yvette), p. 467.

Levison, H. 1987. Ap.J. 320, L93.

Lynden-Bell, D. 1967. MNRAS 136, 101.

May, A. \& van Albada, T.S. 1984. MNRAS 209, 15.

Mihos, J.C. \& Hernquist, L. 1994a. Ap.J. 431, L9.

Mihos, J.C. \& Hernquist, L. 1994b. Ap.J. 437, L47.

Monaghan, J.J. 1992. ARA\&A 30, 543.

Negroponte, J. \& White, S.D.M. 1983. MNRAS 205, 1009.

Nelson, R.P. \& Papaloizou, J.C.B. 1994. MNRAS 270, 1.

Noguchi, M. 1988. A\&A 203, 259.

Noguchi, M. 1991. MNRAS 251, 360.

Sanders, D.B. et al. 1988. Ap.J. 324, L55.

Schweizer, F. 1982. Ap.J. 252, 455.

Schweizer, F. 1986. Science 231, 227.

Scoville, N.Z. et al. 1991. Ap.J. 366, L5.

Simkin, S.M., Su, H.J. \& Schwarz, M.P. 1980. Ap.J. 237, 404.

Steiman-Cameron, T.Y., Kormendy, J. \& Durisen, R.H. 1992. A.J. 104, 1339.

Stockton, A. 1974. Ap.J. 187, 219.

Toomre, A. \& Toomre, J. 1972. Ap.J. 179, 623. 
Toth, G. \& Ostriker, J.P. 1992, Ap.J. 389, 5.

Urdy, S. 1993. $A \& A$ 268, 35.

Weil, M.L. \& Hernquist, L. 1994. Ap.J. 431, L79.

White, S.D.M. 1978. MNRAS 184, 185.

White, S.D.M. 1980. MNRAS 191, 1P.

White, S.D.M. 1982. In "Morphology and Dynamics of Galaxies", eds. L. Martinet \& M. Mayor (Geneva Observatory: Sauverny), p. 291.

White, S.D.M. \& Rees, M.J. 1978. MNRAS 183, 341.

\section{Discussion}

Khachikian: I have a strong point of view: not all unusual, complex galaxies, especially those with complex nuclei, are results of merging. It is necessary to be careful. As an example you showed NGC 520 which is known as an Irr galaxy of the M 82 type and was studied in detail by many authors. Barnes: I too would resist the tendency to label all galaxies with complex or unusual nuclear regions as mergers. But in NGC 520 the diagnosis is based not on the nuclear regions, but on outer features - including what seem to be tidal tails. It is very hard to explain such nonequilibrium features in an old stellar population without invoking a large-scale time-dependent gravitational field. Since NGC 520 has no nearby companions which could produce such a field, I believe this galaxy is merging.

Khachikian: I would like to ask a question more philosophical than astrophysical: do you first assume that a given galaxy is a merger, and then study it, or do you first study it and then conclude that it is a merged galaxy?

Barnes: If one studies a galaxy and finds that it has features characteristic of a merger, then merging is a reasonable hypothesis - to be accepted or rejected on the basis of further study.

Windhorst: Two questions: 1 . What would change in your simulations if you added a $10^{9} \mathrm{M}_{\odot}$ point source (AGN) to the center of each galaxy before their approach? 2. In ultradeep HST images, various groups have seen "tadpole"-like objects. Cowie et al. (1995 preprint) call them "chaingalaxies" and suggest they may be spirals in formation. To me they seem to be more dynamically disturbed objects in groups or perhaps distant clusters. What can they be dynamically? Can they form normal spirals?

Barnes: 1. I think the simulations may still be too crude to reliably show the dynamical effects of even $10^{9} \mathrm{M}_{\odot}$ black holes. 2 . I doubt that chaingalaxies can collapse to form normal disks - too much scope for angular momentum transport. 